\title{
Hemodynamic Considerations in the Pathophysiology of Diabetic Peripheral Neuropathy
}

\author{
Daryl I Smith* and Hai T Tran \\ Department of Anesthesiology and Perioperative Medicine, University of Rochester, USA
}

Submission: January 27, 2018; Published: February 07, 2018

*Corresponding author: Daryl I Smith, Department of Anesthesiology and Perioperative Medicine University of Rochester, Rochester, NY, USA, Tel: (585)-276-3770; Fax: (585)-244-7271; Email: daryl_smith@umrc.rochester.edu

\begin{abstract}
Diabetic peripheral neuropathy (DPN) presents one of the greatest on-going challenges to both acute and chronic pain management yet our understanding of the origins and pathogenesis of this complex disease state is inadequate. This review will discuss some of the more conclusive literature regarding DPN as impacted by hemodynamic alterations. Because of the multifaceted origins of diabetic peripheral neuropathy, this cannot be discussed as a single entity but we can seek to identify a final common pathway.

The vascular supply to peripheral nerves is often overlooked in the natural history of common, well-understood diseases as well as in the management of acute and chronic pain syndromes. When chronic peripheral neuropathic pain develops, the results can be devastating with significant impairment in quality of life (QoL) functioning in the activities of daily living (ADL) and cause significant loss of productivity. We will examine some of the known pathogenetic origins of DPN, and then discuss the effect of resultant hemodynamic alterations to determine any correlations between these alterations and specific effects upon neural structure and function in the hyperglycemic milieu. Lastly, we proffer some of the data for newer potential treatments of DPN.
\end{abstract}

Keywords: Diabetic peripheral neuropathy; Hemodynamics; Diabetes; Neural injury

\section{Introduction}

Peripheral neuropathy (PN) of all types is a relatively rare but well-known degenerative disorder of the peripheral nervous system with an estimated annual incidence of 1.6 per 100000 and a prevalence of 2.4 percent in the United States [1,2]. For persons forty years and older, the prevalence is about five-fold higher (11.5 percent) and 10 times higher in diabetic individuals (21.2 percent) [3]. Among the causes of PN are caused by diabetes mellitus, toxins, alcohol abuse, or paraneoplastic syndromes with diabetes mellitus as the most common cause of PN worldwide. Both sensory and/or motor component (sensorimotor) of the peripheral nervous system (PNS) can be affected. The symptoms, severity and duration of PN depend on the type of nerve affected, sensory, motor or both, the inciting incidence or causative agent, and the length of exposure. Motor neuropathy is characterized by muscular weakness affecting mobility, coordination, and respiratory function. Sensory neuropathy is characterized by pain, numbness, burning sensation, absent or diminished reflexes and sensation to touch.
The generalized category DPN is a common neurological manifestation of both Type 1 and Type 2 diabetes. It affects up to $50 \%$ of diabetics and there is an even greater incidence in those with subclinical manifestations, i.e., asymptomatic DPN. The PN can involve both motor and sensory nerves and the complexity of the metabolic and vascular factors involved still has not been fully elucidated. The sensory loss is classically described as a "stocking and glove distribution" involving both hands and legs. The underlying pathology causing this neuropathy appears to involve both macro- and microvascular processes. We now know that diabetic neuropathy is not likely one separate or discreet entity but, rather, a malady that is the endpoint of a number of different mechanisms that may be enhanced by the hyperglycemic state.

The problem we encounter as practitioners who must manage the care of patients suffering from DPN and, more specifically, the debilitating pain that results from DPN is that the categorical treatments available for the management of this malady are oft 


\section{Current Research in Diabetes \& Obesity Journal}

times ineffective. Standard, accepted treatments have included tricyclic antidepressants, serotonin-norepinephrine reuptake inhibitors, or $\gamma$-aminobutyric acid analogues gabapentin and pregabalin. Patients in whom these initial regimen agents are ineffective oftenreceive opioids and topical therapies such as capsaicin as the next line of treatment. Unfortunately, these therapies lack the desired consistent efficacy necessary to instill confidence in either physicians or patients that adequate analgesia can routinely be obtained.

The relationship of DPN to regional neural blood flow was asserted by Dillon et al. $[4,5]$ when they concluded that slowhealing of neuropathic ulcers were associated with a loss of cholinergic nerve function and that cholinergic stimulation would increase capillary blood flow. They also suggested that improved blood flow to the neural supply of the region may have an overall beneficial effect to the insulted tissue [4]. In 1997, the same group advanced their work to conclude that peripheral blood flow is inversely related to the degree of peripheral neuropathy [5]. These important hemodynamic dependent mechanisms of neural injury now appear to stem from galactose neuropathy and resultant oxidative stress; angiotensin converting enzymemediated neuropathy; glycochelate and transition metal mediated neuropathy.

\section{Pathophysiology}

Galactose neuropathy and oxidative stress: As far back as 1984 galactose was implicated as a cause for PN in a murine, diabetic model. Using C14iodoantipyrine as a radioactive tracer of tissue perfusion, Myers et al. [6] noted a significant decline in nerve blood flow in animals that had ingested galactose for 6 months versus controls. There was a positive correlation between galactose ingestion, endoneurial edema, increased tissue pressure, and ultimate demyelination of nerve fibers. The group also found that Schwann cells showed significant glycogen accumulation in regions in which there was edema. This bolstered the argument that edema, rather than neural hyperactivity in the sorbitol pathway was responsible for the pathological changes in galactosemic neuropathy [6]. Most recently however the sorbitol pathway has returned to the fore as the primary mechanism of glycemic based neural injury. The sorbitol pathway is an alternative to a primary intracellular pathway of glucose metabolism; the polyol pathway. The polyol pathway is dependent upon aldose reductase (AR) which reduces toxic aldehydes in the cell to inactive alcohols. In the hyperglycemic state AR is rendered incapable dispatching the glycemic burden in the usual fashion and thus transitions to the reduction ofglucose to sorbitol. The glucose reduction pathway which generates sorbitol also subsequently oxidizes sorbitol to fructose. The sorbitol branch of the polyol pathway consumes the cofactor NADPH, which is the essential cofactor for regenerating the important intracellular antioxidant, reduced glutathione (GSH). Lack of GSH has been shown to induce as well as exacerbate oxidative stress. The result of which includes thickening of the capillary basement membrane in the retina, kidney and muscle [7]. The current thought regarding diabetesspecific microvascular disease in the vasa nervorum includes the following: hyperglycemia causes abnormalities in blood flow and increased vascular permeability. Early discussions of diabetic neuropathy have emphasized the hyperglycemic insult. The key factors of emphasis are resistance to vasodilators, hypersensitivity to vasoconstrictors and elaboration of permeability factors such as VEGF. The sum of these changes results in edema, ischemia and hypoxia-induced axonal degeneration in peripheral nerves [7]. In 2016, Ozaki et al. [8] sought to examine the role of hypertension in diabetic neuropathy. They used a murine model using alloxan-induced diabetic rats and non-diabetic rats. In both groups blood pressure was maintained above $140 \mathrm{mmHg}$. While both groups showed endothelial hypertrophy and vessel lumen narrowing, more severe duplication of the basal lamina surrounding the endothelium and pericytes of the endoneurial vessels was reported [8].

Angiotensin converting enzyme (ACE) mediated neuropathy: The possible link between vasoactivity and ACE in DPN (at least in the murine model) was asserted in a 2007 study by Wang et al. [9] In this study, serum levels of the adipokine resistin was shown to correlate with systolic blood pressure, diastolic blood pressure and serum epithelin levels, and to negatively correlate with the potent vasodilator, nitric oxide [9]. In a more recent study, resistin was shown to induce hypertension and insulin resistance in wild type mice believed to occur by the upregulation of angiotensin (Agt) toll-like receptor 4 expression [10]. In toll-like receptor 4 (tlr4)-negative mice or in mice treated with the angiotensin-converting enzyme inhibitor, perindopril, resistin had no effect. The authors concluded from this that resistin activates the renin-NF angiotensin system via the TLR4/P65-NFKB subunit/Agt pathway which links insulin resistance to hypertension. The higher serum resistin levels in patients with diabetic neuropathy versus diabetics without peripheral neuropathy suggests that resistin may play a role in the pathogenesis of type 2 diabetes and diabetic peripheral neuropathy. The question is also raised regarding whether hypertension secondary to resistin is a causative factor in this neuropathy [10].

Transition metal mediated neuropathy-glycochelates: The role of transition metals was argued in a review article by Qian et al. [11]. They presented data that heavily glycated proteins, known to accumulate in individuals suffering from diabetes, gain an increased affinity for transition metals such as iron and copper. This affinity results in the accumulation of bound metal by elastin and collagen within the arterial wall. The bound metal is believed to cause the catalytic destruction of endothelium-derived releasing factor (nitric oxide or a nitric oxide derivative). The loss of vasodilatory ability (or chronic vasoconstriction) impairs blood to peripheral nerves with resultant deprivation of oxygen and critical nutrients. The authors cited initial studies that suggest the administration of 


\section{Current Research in Diabetes \& Obesity Journal}

chelators such as desferrioxamine may prevent or reverse slower peripheral nerve conduction and neuronal blood flow [11].

\section{Therapeutics}

Genetic treatment of hemodynamic derangements caused by DPN: Reversal of experimental diabetic neuropathy in a murine model induced by two different techniques was explored by Schratzberger et al. [12]. Both streptozotocin- and alloxan- induced diabetes models were employed and nerve blood flow was assessed by laser Doppler imaging or direct detection of a locally administered fluorescent lectin. In both models, intramuscular gene transfer of plasmid DNA encoding VEGF-1 or VEGF-2 resulted in increases in vascularity and nerve blood flow to levels found in control animals. The group also reported that constitutive over-expression of both transgenes resulted in restoration of large and small fiber peripheral nerve function as measured by motor and sensory nerve conduction velocities. Similar findings in a lapine model are also reported. There is accumulating evidence, then, that genetic therapy may have a role in the treatment of peripheral neuropathy of diabetic origin. Unlike the observed efficacy of this gene therapy in chemotherapy-induced neuropathy, considerations for induction of related angiogenesis would not be a factor in the decision to institute plasmid DNA therapy; however, a concern for possible retinal angiogenesis and a question of initiating or worsening diabetic retinopathy may be a concern. In this regard further research is needed first to assess the associated angiogenicity of this treatment in animals and second to establish whether any benefit of this de facto genetic hemodynamic therapy can be extrapolated to a human model [12].

Endothelium-dependent and endothelium-independent micro-vasodilatation therapy: The role of endotheliumdependent and endothelium-independent micro-vasodilatation and their relationships to neural microcirculatory control was examined in Type I and Type II diabetic patients by Kilo et al. [13]. They used iontophoresis of acetylcholine and nitroprusside in a dose-response technique to elicit C-fiber mediated vasodilation. As expected, endothelium-dependent vasodilation of the cutaneous microcirculation was attenuated in Type II diabetic subjects versus control; however, there was no significant difference between the endothelium-dependent vasodilation in Type I diabetics versus controls. There was no difference between either diabetic group (Type I or Type II) regarding endothelium-independent vasodilation. They also found that the C-fiber- mediated axon reflex was impaired in both Type I and Type II diabetics, which the group stated was consistent with a small fiber neuropathy. The study led to the conclusion that endothelial function and nitric oxide play a significant role in the pathogenesis of PN in Type II diabetic patients and that this disease process is the result in part of significant C-fiber impairment. Again, the function of C-fibers, the neural component of peri-neural hemodynamics, and the peri-neural chemical milieu may begin to suggest a common pathway for the perfusion of peripheral nerves and the development of PN.

ACE inhibitor therapy: Angiotensin-converting enzyme (ACE) inhibition was considered as early as 1998 as playing a role in the treatment of human diabetic neuropathy in a randomized trial. In this work 41 patients with normotension, "mild" diabetic neuropathy and a diagnosis of type I or type II diabetes were placed in the randomized double-blind placebo-controlled trial. In which the experimental group was treated with an ACE inhibitor. Assessments of treatment efficacy were made using the endpoint of neuropathic symptoms, deficit scores, vibratory perception threshold, peripheral-nerve electrophysiology, and cardiovascular autonomic function at 6 and 12 months of treatment with the primary endpoint of change in peroneal motor nerve conduction velocity. The study revealed a significant increase in peroneal motor nerve conduction velocity, and M-wave amplitude and sural nerve action potential amplitude. However, vibration-perception threshold, autonomic function and the symptoms of neuropathy and deficit score showed no improvement in either group. Yet the question remained whether reversal of neural functional impairment can ultimately lead to symptomatic improvement and further clinical study was needed to make this determination [14].

Free radicals scavenging therapy: The oxidative stress and pro-inflammatory processes which contribute to vascular complications including endothelial dysfunction and peripheral neuropathy in diabetes mellitus was examined in a 2006 study by Nangle et al. [15]. In this work the group administered eugenol -which is known to have antioxidant and anti-inflammatory properties especially in the inhibition of lipid peroxidation- to streptozotocin induced diabetic rats [16]. The group analyzed endoneurial blood flow reduction; gastric fundus maximum nitrergic nerve-mediated relaxation reduction; and maximum endothelium-dependent relaxation reduction in renal artery rings all in diabetic animals. Eugenol significantly improved or completely reversed each of these reductions but did not affect diabetes-increased sensitivity to phenylephrine-mediated contraction. Nevertheless, the study demonstrated that both vascular as well as neural complications of experimental diabetes are improved by the antioxidant/anti-inflammatory agent eugenol and reinforces the argument for the role of pressure-flow perfusion dependence on the development of oxidative stress-related PN.

In $2006 \mathrm{Li}$ et al. [17] examined the role of antioxidant therapy in the treatment of diabetic neuropathy in a murine model. The group looked at the effect of the antioxidant taurine upon sensory nerve conduction velocity, nerve blood flow and sensory threshold in hyperglycemic, Zucker diabetic fatty (ZDF) rats. They established experimental groups consisting of lean, non-diabetic rats (ND); ND rats treated with taurine; untreated ZDF diabetic rats (D); and D rats treated with taurine. When compared with controls, deficits in nerve conduction, velocity (both motor and sensory) and nerve blood flow were 


\section{Current Research in Diabetes \& Obesity Journal}

all reversed by taurine. They concluded that antioxidant therapy may be useful in the treatment of experimental Type II diabetes [17]. The role of peroxynitrite-mediated nitrosative stress in the development of diabetic neuropathy was studied in a murine model by Negi et al. [18]. In this work the effect of a combination of peroxynitrite decomposition catalyst (PDC), FeTMPyP [15] and a poly (ADP-ribose) polymerase (PARP) - a nuclear enzyme activated after detection of DNA damage - inhibitor, 4-ANI. The rationale for the use of the PARP inhibitor was the role that overactivation of this enzyme is believed to play in the development of diabetic neuropathy [19]. The group studied the following endpoints: motor conduction velocity and nerve blood flow for evaluating neural function; malondialdehyde and peroxynitrite levels to detect oxidative stress-nitrosative stress; and nicotinamide adenine dinucleotide (NAD+) concentration in sciatic nerve to assess NAD+ overproduction of PARP. Treatment with combination of FeTMPyP and 4-ANI led to improvement in neural function and also attenuated the oxidative-nitrosative stress markers. The combination also reduced the overactivation of PARP which was demonstrated by increased levels of NAD+ and by the demonstration of decreased PARP immunopositivity in sciatic nerve microsections. The authors concluded that treatment with a combination of a PDC and a PARP inhibitor attenuates alterations in peripheral nerves in experimental diabetic neuropathy.

The role of melatonin in the limiting of oxidative stressrelated neural injury was examined by Manasaveena [20]. In this work, the group used streptozotocin-induced diabetic neuropathy, and oxaliplatin-induced sensory neuropathy murine models. They administered melatonin at doses of $3 \mathrm{mg} /$ $\mathrm{kg}$ and $10 \mathrm{mg} / \mathrm{kg}$ to the diabetic animals during the $7^{\text {th }}$ and $8^{\text {th }}$ week after diabetes induction. They administered the same doses of melatonin daily to the oxaliplatin treated animals. In both models, melatonin administration significantly increased tail flick latency-an indication of reduced hypersensitivity- when compared to control animals. Motor nerve conduction and nerve blood flow were improved in melatonin treated diabetic animals. Of particular significance and interest, lipid peroxidation was also improved by melatonin in both experimental models. The group speculated that since oxidative stress plays a major role in peripheral neuropathy induced both in the setting of diabetic as well as chemotherapeutic neuropathies, that melatonin might serve as a possible therapeutic choice because of its antioxidant and anti-inflammatory activity. The role of oxidative stress in the process of neurodegenerative diseases and other syndromes was reinforced in an earlier review in 2002 by Srinivasan [21] in which emphasis was placed upon the scavenging of hydroxyl carbonate and various organic radicals; and peroxynitrite and other reactive nitrogen species, in addition to melatoninmedicated stimulation of superoxide dismutase, glutathione peroxidase and glutathione reductase. These works strongly suggest that the impact of oxidative stress upon the function of the neural microvasculature warrants closer study especially with emphasis processes with antioxidant therapy. Another argument for the clinical use of antioxidant therapy was examined by Pop-Busui et al. [22]. The main emphasis of his work was on measures of cardiovascular autonomic neuropathy (CAN) and myocardial blood flow and it was performed as a randomized parallel, placebo-controlled trial. Participants were evaluated by cardiovascular autonomic reflex testing positron emission tomography and adenosine stress testing. Markers of oxidative stress included 24 hour urinary-F2-isoprostanes. Diabetic peripheral neuropathy was evaluated by symptoms, signs, electrophysiology, and intra-epidural nerve fiber density. The study participants underwent a 24-month intervention consisting of anti-oxidant treatment with allopurinol, alphalipoic acid and nicotinamide or placebo. They found that in the cohort of type 1diabtes with mild-to-moderate CAN, a combination antioxidant treatment regimen did not prevent progression of CAN; had no beneficial effects on myocardial perfusion or the measures of diabetic peripheral neuropathy.

The need for further clinical study of antioxidant therapy, particularly in the targeting of lipid peroxidation in diabetic peripheral neuropathy as well as in other peripheral neuropathic subtypes is strongly indicated [22]. In 2014 Zhang et al. [23] evaluated the effect of salvianolic acid on diabetic peripheral neuropathy [23]. They analyzed peripheral electrophysiology, hemodynamics in the peripheral microcirculation, ultrastructure of sciatic nerve observation, and biochemical indicators. They found down regulation of malonyldialdehyde, an end product of lipid peroxidation; advanced glycation end products; total cholesterol; plasma triglycerides; and a decreasing tendency of fructosamine, a glycated protein used as a monitor of glycemic control. In addition they found up regulation of neural nitric oxide synthase (nNOS); brain derived neurotrophic factor which is decreased when blood glucose levels are elevated [24]; and glial cell line-derived neurotrophic factor which has been shown to reverse the neuronal loss caused by a reduction in Aktmediated survival signaling [25]. They concluded that salvianolic acid could improve peripheral nerve function in type 2 diabetic rats by decreasing oxidative stress damage, improving vascular function in the peripheral microcirculation, and that the drug could promote the expression of neurotrophic factors.

The antiatherosclerotic and anti-inflammatory effects of the cannabinoid, rimonabant, on peripheral neuropathies in the diabetic murine model have been examined in a recent work. In this study diabetes was induced in rats using streptozotocin and the animals were treated for 24 weeks with $10 \mathrm{mg} / \mathrm{kg} /$ day rimonabant or placebo. The group then quantified the densities of intraepidermal nerve fibers and total skin capillary length. They also measured current threshold, skin blood flow after treadmill running and TNF- $\alpha$ level in spinal cord tissue or plasma. The group found that rimonabant treatment significantly improved the decreased intraepidermal nerve fiber density and alleviated the increased current perception threshold in rimonabant treated versus control diabetic rats. The group reported that 
the responses were clearly associated with the attenuation of skin capillary loss, increase in skin blood flow, and reduction in tissue TNF- $\alpha$ levels. The group concluded that their findings suggested that rimonabant may be beneficial in the treatment of experimental diabetic peripheral neuropathy possibly due to its micro- and macrovascular protective effects [26].

\section{Conclusion}

At this juncture there have been several promising approaches that have specifically targeted mechanisms involved in the development of the neuropathy. These projected approaches include restoration of insulin production capacity (pancreatic transplantation) [27]; free radical scavenging with the use of antioxidants [28]; inhibition of the glycol pathway [29]; thiamine/ vitamin B1 analogues to inhibit pathways involved in vascular damage associated with diabetes [30]; and manipulation of $\mathrm{Na}+\mathrm{K}+$ ATPase and a variety of transcription factors [31]; ACE inhibitors; and exploiting neuroprotective effects of the hydroxymethyl glutaryl CoA reductase inhibitors which control serum lipid levels [32]. It is evident from these approaches that all but two (thiamine/vitamin B1 analogues and ACE inhibitors specifically address the role of pressure-flow relationships in the vaso nervorum in the treatment of DPN. These relationships clearly need to be evaluated both in vitro as well as in vivo in human subjects in order to explore possible means of enhancing the treatment of peripheral neuropathy and controlling progression. The evidence of a final common pathway of impaired microvascular reactivity at the vasa nervorum level may be of paramount importance as new therapies are sought to limit the effect of this devastating neuropathy.

\section{References}

1. Azhary H, Farooq MU, Bhanushali M, Majid A, Kassab MY (2010) Peripheral neuropathy: differential diagnosis and management. American family physician 81(7): 887-892.

2. Laughlin RS, Dyck PJ, Melton LJ, Leibson C, Ransom J, et al. (2009) Incidence and prevalence of CIDP and the association of diabetes mellitus. Neurology 73(1): 39-45.

3. Cheng YJ, Gregg EW, Kahn HS, Williams DE, De Rekeneire N, et al. (2006) Peripheral insensate neuropathy--a tall problem for US adults? Am J Epidemiol 164(9): 873-880.

4. Dillon RS (1991) Role of cholinergic nervous system in healing neuropathic lesions: preliminary studies and prospective, doubleblinded, placebo-controlled studies. Angiology 42(10): 767-778.

5. Dillon RS (1997) Patient assessment and examples of a method of treatment. Use of the circulator boot in peripheral vascular disease. Angiology 48(5 Pt 2): S35-S58.

6. Myers RR, Powell HC (1984) Galactose neuropathy: impact of chronic endoneurial edema on nerve blood flow. Ann Neurol 16(5): 587-594.

7. Brownlee M (2001) Biochemistry and molecular cell biology of diabetic complications. Nature 414(6865): 813-820.

8. Ozaki K, Hamano H, Matsuura T, Narama I (2016) Effect of deoxycorticosterone acetate-salt-induced hypertension on diabetic peripheral neuropathy in alloxan-induced diabetic WBN/ Kob rats. J Toxicol Pathol 29(1): 1-6.
9. Wang XH, Wang DQ, Chen SH, Zhang L, Ni YH (2007) The relationship between resistin and the peripheral neuropathy in type 2 diabetes. Zhonghua Yi Xue Za Zhi 87(25): 1755-1757.

10. Jiang Y, Lu L, Hu Y, Li Q, An C, et al. (2016) Resistin induces hypertension and insulin resistance in mice via a TLR4-Dependent Pathway. Sci Rep 6: 22193.

11. Qian M, Eaton JW (2000) Glycochelates and the etiology of diabetic peripheral neuropathy. Free Radic Biol Med 28(4): 652-656.

12. Schratzberger P, Walter DH, Rittig K, Bahlmann FH, Pola R, et al. (2001) Reversal of experimental diabetic neuropathy by VEGF gene transfer. J Clin Invest 107(9): 1083-1092.

13. Kilo S, Berghoff M, Hilz M, Freeman R (2000) Neural and endothelial control of the microcirculation in diabetic peripheral neuropathy. Neurology 54(6): 1246-1252.

14. Malik RA, Williamson S, Abbott C, Carrington AL, Iqbal J, et al. (1998) Effect of angiotensin-converting-enzyme (ACE) inhibitor trandolapril on human diabetic neuropathy: randomised double-blind controlled trial. Lancet 19-352(9145): 1978-1981.

15. Nangle MR, Gibson TM, Cotter MA, Cameron NE (2006) Effects of eugenol on nerve and vascular dysfunction in streptozotocin-diabetic rats. Planta Med 72(6): 494-500.

16. Gulcin I (2011) Antioxidant activity of eugenol: a structure-activity relationship study. J Med Food 14(9): 975-985.

17. Li F, Abatan OI, Kim H, Burnett D, Larkin D, et al. (2006) Taurine reverses neurological and neurovascular deficits in Zucker diabetic fatty rats. Neurobiol Dis 22(3): 669-676.

18. Negi G, Kumar A, Sharma SS (2010) Concurrent targeting of nitrosative stress-PARP pathway corrects functional, behavioral and biochemical deficits in experimental diabetic neuropathy. Biochemical and biophysical research communications. 391(1): 102-106.

19. Pacher P, Szabo C (2005) Role of poly(ADP-ribose) polymerase-1 activation in the pathogenesis of diabetic complications: endothelial dysfunction, as a common underlying theme. Antioxid Redox Signal 7(11-12): 1568-1580.

20. Manasaveena A, Veera Ganesh Y, Reddemma S, Manish Kumar J, Naidu VGM, et al. (2013) Evaluation of neuroprotective effect of melatonin in animal models of peripheral neuropathy induced by streptozotocin and oxaliplatin. Indian Journal of Pharmacology 45: S238-S239.

21. Srinivasan V (2002) Melatonin oxidative stress and neurodegenerative diseases. Indian J Exp Biol 40(6): 668-679.

22. Pop Busui R, Stevens MJ, Raffel DM, White EA, Mehta M, et al. (2013) Effects of triple antioxidant therapy on measures of cardiovascular autonomic neuropathy and on myocardial blood flow in type 1 diabetes: a randomised controlled trial. Diabetologia. 56(8): 18351844.

23. Zhang L, Yu X, Yang X, Huang Z, Du G (2014) Effect of salvianolic acid A on diabetic peripheral neuropathy in type 2 diabetic rats. Basic and Clinical Pharmacology and Toxicology 115:114.

24. Krabbe KS, Nielsen AR, Krogh Madsen R, Plomgaard P, Rasmussen P, et al. (2007) Brain-derived neurotrophic factor (BDNF) and type 2 diabetes. Diabetologia 50(2): 431-438.

25. Anitha M, Gondha C, Sutliff R, Parsadanian A, Mwangi S, et al. (2006) GDNF rescues hyperglycemia-induced diabetic enteric neuropathy through activation of the PI3K/Akt pathway. J Clin Invest 116(2): 344356.

26. Liu WJ, Jin HY, Park JH, Baek HS, Park TS (2010) Effect of rimonabant, the cannabinoid $\mathrm{CB} 1$ receptor antagonist, on peripheral nerve in streptozotocin-induced diabetic rat. Eur J Pharmacol 637(1-3): 70-76. 


\section{Current Research in Diabetes \& Obesity Journal}

27. Agudo R, Valls Sole J, Recasens M, Fabregat N, Ricart MJ, et al. (2002) Effects of kidney and pancreas transplantation on neurophysiological rates of polyneuropathy and autonomous cardiac function in diabetic patients with end-stage renal disease. Med Clin (Barc) 118(14): 534538.

28. Chong MS, Hester J (2007) Diabetic painful neuropathy: current and future treatment options. Drugs 67(4): 569-585. Hotta N, Akanuma Y, Kawamori R, Matsuoka K, Oka Y, et al. (2006) Long-term clinical effects of epalrestat, an aldose reductase inhibitor, on diabetic periphera neuropathy: the 3-year, multicenter, comparative Aldose Reductase Inhibitor-Diabetes Complications Trial. Diabetes care 29(7): 15381544 .

29. Hotta N, Akanuma Y, Kawamori R, Matsuoka K, Oka Y, et al. (2006) Longterm clinical effects of epalrestat, an aldose reductase inhibitor, on diabetic peripheral neuropathy: the 3-year, multicenter, comparative Aldose Reductase Inhibitor-Diabetes Complications Trial. Diabetes care 29(7): 1538-1544.

30. Hammes HP, Du X, Edelstein D, Taguchi T, Matsumura T, et al. (2003) Benfotiamine blocks three major pathways of hyperglycemic damage and prevents experimental diabetic retinopathy. Nat Med 9(3): 294299.

31. Cotter MA, Ekberg K, Wahren J, Cameron NE (2003) Effects of proinsulin C-peptide in experimental diabetic neuropathy: vascular actions and modulation by nitric oxide synthase inhibition. Diabetes 52(7): 1812-1817.

32. Fried LF, Forrest KY, Ellis D, Chang Y, Silvers N, et al. (2001) Lipid modulation in insulin-dependent diabetes mellitus: effect on microvascular outcomes. J Diabetes Complications 15(3): 113-119.

\begin{tabular}{l} 
Your next submission with Juniper Publishers \\
will reach you the below assets \\
- Quality Editorial service \\
- Swift Peer Review \\
- Reprints availability \\
- E-prints Service \\
- Manuscript Podcast for convenient understanding \\
- Global attainment for your research \\
- Manuscript accessibility in different formats \\
( Pdf, E-pub, Full Text, Audio) \\
- Unceasing customer service \\
Track the below URL for one-step submission \\
https://juniperpublishers.com/online-submission.php \\
\hline
\end{tabular}

